\title{
Supporting information for: Toward understanding optical properties of amyloids: a reaction path and nonadiabatic dynamics study
}

Luca Grisanti * 1,3, Marin Sapunar ${ }^{2}$, Ali Hassanali ${ }^{3}$, and Nađa Došlić ${ }^{\dagger} 2$

${ }^{1}$ Division of Theoretical Physics, Ruđer Bošković Institute, Bijenička cesta 54, 10000 Zagreb, Croatia

${ }^{2}$ Department of Physical Chemistry, Ruđer Bošković Institute, Bijenička cesta 54, 10000 Zagreb, Croatia

${ }^{3}$ Condensed Matter and Statistical Physics, International Centre for Theoretical Physics, Strada Costiera 11, Trieste 34151 Italy

\section{Contents}

S1 Computational details $\quad$ S2

S1.1 Model systems . . . . . . . . . . . . . . . . . . . . . . S2

S1.2 Methods . . . . . . . . . . . . . . . . . . . . . . . . S2

S1.2.1 Ground state dynamics . . . . . . . . . . . . . . . . . . S2

S1.2.2 Static calculations . . . . . . . . . . . . . . . . . . . . S3

S1.2.3 Nonadiabatic dynamics simulations and fluorescence spectra . . . . . . S4

S2 Results and analysis $\quad$ S6

S2.1 Excitation energies and dynamics simulations . . . . . . . . . . . . S6

S2.2 Structural fluctuations and the role of termini $\ldots \ldots \ldots \ldots$. . . . . . S14

$\begin{array}{ll}\text { S3 Cartesian coordinates of the reference 2B7(C) structure } & \text { S17 }\end{array}$ 


\section{S1 Computational details}

\section{S1.1 Model systems}

The prototype model system 2B7 was obtained starting from available crystal structure of hexapeptide AIIGLM, registered in the PDB database as $2 \mathrm{Y}^{3} \mathrm{~J}^{1}$ and corresponding to amyloidic sequence $\mathbf{A} \beta_{30-35}$. To obtain $\mathbf{2 B 7}$, the two chains were taken from two parallel $\beta$-sheet chains, keeping the terminus $\mathrm{COOH}$ as in the deposited structure. In this $\mathrm{COOH}$-containing chain, 4 carbonyl groups are present in total, and the opposite site was capped with a $\mathrm{CHO}$ group. In the second chain of $\mathbf{2 B 7}$, three carbonyls groups were kept. One end was converted to an amide group and the other contains an $-\mathrm{NH}_{2}$ terminus. Besides, we dropped any sidechains and replaced with hydrogens, e.g. our prototypical non-termini amino acidic unit is glycine (see Figure 1). This choice offers the good combination of computational feasibility and generality, since the most important chemical ingredients were kept. This particular configuration is the reference configuration $2 \mathbf{B 7}(\mathbf{C})$. The other model systems were obtained starting from the above by: a) consistently extending both chains for 2B11 b) adding a replica of the $\mathrm{COOH}$ containing chain for 3B11. Static calculations have been performed only for the parallel $\beta$-sheet arrangement, but nonadiabatic dynamics simulations were run also for analogous structures obtained from anti-parallel $\beta$-sheet.

\section{S1.2 Methods}

\section{S1.2.1 Ground state dynamics}

For all the model systems 2B7, 2B11 and 3B11, we performed in vacuum ground state (gs) geometry optimizations, and starting from these structures we run ab initio molecular dynamics (aiMD) for the ground state. In aiMD, ab initio Born-Oppenheimer MD is used for the propagation of classical nuclei, and this has proven to be a fast and reliable computational frame according to our experience. ${ }^{2} \mathrm{All}$ ground state calculations were run using the CP2K package. ${ }^{3}$ A convergence criterion of $5 \times 10^{-7}$ a.u. was used for the optimization of the wave function. Using the Gaussian and plane wave methods, the wave function was expanded in the Gaussian double- $\zeta$ valence polarized (DZVP) basis set. An auxiliary basis set of plane waves was used to expand the electron density up to a cutoff of 320 Ry. For the simulations we used the 
gradient correction to the local density approximation and Goedecker-Teter-Hutter (GTH) pseudopotentials for treating the core electrons. ${ }^{4}$ Unless stated otherwise, all calculations employed the Becke-Lee-Yang-Parr (BLYP) ${ }^{5,6}$ functional with the D3(0) Grimme dispersion corrections for van der Waals interactions. ${ }^{7}$ Geometry minimizations were performed using the BroydenFletcher-Goldfarb-Shanno (BFGS) minimizer in CP2K. ${ }^{8}$ The aiMD simulations of the ground state were performed using the Quickstep algorithm ${ }^{9}$ which is part of the CP2K package. ${ }^{3}$ The finite temperature simulations were conducted within the canonical NVT ensemble using the canonical-sampling velocity-rescaling thermostat method. ${ }^{10}$ All aiMD simulations were run starting from the minimum without any constraints, hence allowing the systems to explore the ground state potential energy surface around the respective minima. The aiMD simulation time was 9.8 ps for 2B7, 3.4 ps for 2B11 and 3.7 ps for 3B11.

\section{S1.2.2 Static calculations}

The algebraic diagrammatic construction of second order (ADC(2)) method was used for excited-state calculations. ${ }^{11,12}$ Unless stated otherwise, the cc-pVDZ basis set was employed in all ADC(2) calculations. ${ }^{13}$ To describe Rydberg excited states we have used the spincomponent scaled version of $\operatorname{ADC}(2)$ (SCS-ADC(2)) and the aug-cc-pVDZ basis set. ${ }^{14,15}$ Calculations were performed using the Turbomole program package. ${ }^{16,17}$

For the computation of UV absorption spectra of 2B7, 2B11 and 3B11 and the density of excited states (DoES) initial conditions were uniformly and randomly sampled from groundstate dynamics simulations at the DFT level of theory (see above). The number of electronic states included in the computation depends on the size of the system and range from 16 to 20.

Geometry optimizations of the $7 \mathrm{n} \pi^{*}$ states of $2 \mathrm{~B} 7$ were initiated from the reference $\mathbf{2 B 7 ( C )}$ structure with elongated $\mathrm{C}=\mathrm{O}$ bonds. This stabilizes each of the $\mathrm{n} \pi^{*}$ states as the lowest excited state $\left(S_{1}\right)$ making the optimization possible. Because of the flexibility of $2 \mathrm{~B} 7$, the secondary structure could not be preserved during full geometry optimization and more stable $\gamma$-turn structures were formed. However, due to the highly localized nature of the $\mathrm{n} \rightarrow \pi^{*} \operatorname{transi-}$ tions, it was possible to assume that the minima of the different $n \pi^{*}$ states can be approached by allowing geometry optimization of only the atoms in the region around the photo-excited amide group. The atoms of the amide group, those in the two neighboring groups and in the $\mathrm{H}$-bonded amide group on the opposite strand were relaxed during the constrained opti- 
mizations. The reported adiabatic excitation energies are therefore upper bounds. The only exception was the optimization of the $n_{7} \pi_{7}^{*}$ state with excitation localized on the $(C=O)_{7}$ group. For this state, the $\beta$-strand structure was preserved during the full geometry optimization. Adiabatic excitation energies were computed both at the ADC(2)/cc-pVDZ and SCS-ADC(2)/augcc-pVDZ//ADC(2)/cc-pVDZ levels.

Vertical excitation energies of 2B7 computed with SCS-ADC(2)/aug-cc-pVDZ yielded a $\pi$ Ryd state as the lowest one in energy. The adiabatic excitation energy of the $\pi$ Ryd state was computed at the same level. As the state was only moderately stabilized by geometry optimization and its minimum remained firmly above the $\mathrm{n} \pi^{*}$ minima, it was possible to use the $A D C(2) / c c-p V D Z$ method in subsequent reaction path and nonadiabatic dynamics simulations.

The reaction paths from the Franck-Condon region to the minima of the $\mathrm{n} \pi^{*}$ states were constructed by linear interpolation in internal coordinates. The energy profiles were obtained by $\operatorname{ADC}(2)$ single-point energy calculations along the interpolated paths.

\section{S1.2.3 Nonadiabatic dynamics simulations and fluorescence spectra}

Nonadiabatic trajectory surface hopping molecular dynamics simulations were performed employing Tully's fewest switches surface hopping algorithm. ${ }^{18}$ The required nonadiabatic couplings were computed from wave function overlaps. ${ }^{19,20} \mathrm{~A}$ formal CIS wave function was constructed using the $A D C(2)$ singles amplitudes to evaluate overlaps between wave functions at two time steps of the nuclear dynamics. ${ }^{21-23}$ To efficiently compute overlaps for systems of relatively large size (2B7-3B11) the recently developed method based on the expansion of the CIS wave function in the natural transition orbital basis was employed. ${ }^{20}$ Newton's equations for nuclear motion were integrated in time steps of $0.5 \mathrm{fs}$ using the velocity-Verlet algorithm. Hopping probabilities were evaluated using the locally diabatic formalism of Granucci et al. ${ }^{24}$ The energy-based decoherence procedure of Granucci and Persico with $\alpha=0.1 \mathrm{E}_{h}$ was used. ${ }^{25}$ Our nonadiabatic dynamics code is linked to Turbomole 7.0. ${ }^{17}$

To simulate the nonadiabatic dynamics of 2B11 and 3B11, 4 structures were randomly sampled along the gs aiMD dynamics and vertically excited to the $\mathrm{n} \pi^{*}$ manifold. Trajectories were propagated in the subspace of the electronic ground state and 20 electronically excited states for a total simulation duration of $10-15$ fs.

In the case of 2B7, 21 trajectories, 15 with parallel and 6 with anti-parallel $\beta$-strand arrange- 
ment, were vertically excited to the $\mathrm{n} \pi^{*}$ manifold $\left(\mathrm{S}_{1}-\mathrm{S}_{5}\right)$. Simulations were performed for 250 fs or until the energy gap between the $S_{1}$ and $S_{0}$ states dropped below $0.1 \mathrm{eV}$ which was taken as the time of internal conversion to the ground state. The fluorescence spectrum was simulated starting from $t=15 \mathrm{fs}$, that is, from the time when all trajectories reached the $S_{1}$ state. Vertical emission energies and transition dipole moments $S_{0} \rightarrow S_{1}$ were computed along the nonadiabatic trajectories of parallel 2B7 and then averaged over three time intervals: $15-40$ fs, 40-100 fs, 100-end of trajectory. A standard Gaussian broadening function with parameter $\sigma=0.1 \mathrm{eV}$ was used to produce lineshapes. 


\section{S2 Results and analysis}

\section{S2.1 Excitation energies and dynamics simulations}

Table S1: Spectral assignment, vertical excitation energies (in $\mathrm{eV}$ ) and oscillator strengths $\left(\mathrm{f}_{\text {osc }}\right)$ of the lowest 8 transitions computed at the reference ground state geometry of 2B7(C). Excitations are localized on the terminal amino group $(\pi$ Ryd), 6 amide groups and the terminal carboxyl group. For the numeration of the groups see Figure 1. Calculations were performed at the SCS-ADC(2)/aug-cc-pVDZ level.

\begin{tabular}{lllllllll}
\hline & \multicolumn{8}{c}{ Vertical Excitation Energies $(\mathrm{eV})$} \\
\hline State & $\mathrm{S}_{1}$ & $\mathrm{~S}_{2}$ & $\mathrm{~S}_{3}$ & $\mathrm{~S}_{4}$ & $\mathrm{~S}_{5}$ & $\mathrm{~S}_{6}$ & $\mathrm{~S}_{7}$ & $\mathrm{~S}_{8}$ \\
& $(\pi \mathrm{Ryd})$ & $\left(\mathrm{n}_{5} \pi_{5}^{*}\right)$ & $\left(\mathrm{n}_{1} \pi_{1}^{*}\right)$ & $\left(\mathrm{n}_{7} \pi_{7}^{*}\right)$ & $\left(\mathrm{n}_{3} \pi_{3}^{*}\right)$ & $\left(\mathrm{n}_{4} \pi_{4}^{*}\right)$ & $\left(\mathrm{n}_{2} \pi_{2}^{*}\right)$ & $\left(\mathrm{n}_{6} \pi_{6}^{*}\right)$ \\
\hline$E$ & 4.94 & 5.13 & 5.28 & 5.30 & 5.36 & 5.40 & 5.44 & 5.47 \\
$f_{\text {osc }}$ & 0.0169 & 0.0009 & 0.0007 & 0.0033 & 0.0023 & 0.0012 & 0.0005 & 0.0037 \\
\hline
\end{tabular}

Table S2: Most relevant geometrical parameters of the $7 \mathrm{n} \pi^{*}$ minimum energy geometries of 2B7: the $\mathrm{C}=\mathrm{O}$ bond distance and the pyramidalization angles $\theta_{C}\left(\mathrm{~N}_{i+1} \mathrm{C}_{i}^{\alpha} \mathrm{O}_{i} \mathrm{C}_{i}\right)$ and $\theta_{N}\left(\mathrm{H}_{i}\right.$ $\mathrm{C}_{i}^{\alpha} \mathrm{C}_{i-1} \mathrm{~N}_{i}$ ). For numeration see Figure 1. Only the atoms of the adjacent peptide groups and hydrogen-bonded amide group on the opposite $\beta$-strand were allowed to relax. Calculations were performed at the ADC(2)/cc-pVDZ level.

\begin{tabular}{llllllll}
\hline State & $\mathrm{n}_{1} \pi_{1}^{*}$ & $\mathrm{n}_{2} \pi_{2}^{*}$ & $\mathrm{n}_{3} \pi_{3}^{*}$ & $\mathrm{n}_{4} \pi_{4}^{*}$ & $\mathrm{n}_{5} \pi_{5}^{*}$ & $\mathrm{n}_{6} \pi_{6}^{*}$ & $\mathrm{n}_{7} \pi_{7}^{*}$ \\
\hline $\mathrm{C}=\mathrm{O}$ & 1.44 & 1.45 & 1.45 & 1.47 & 1.45 & 1.46 & 1.46 \\
$\theta_{C}$ & -25.8 & -27.6 & -23.3 & - & -26.9 & -25.0 & -25.9 \\
$\theta_{N}$ & - & -26.6 & -21.2 & -22.0 & - & -26.1 & -26.5 \\
\hline
\end{tabular}


Table S3: Adiabatic excitation energies (in eV) of the $\pi$ Ryd and $7 \mathrm{n} \pi^{*}$ states of $\mathbf{2 B} 7$ computed at the partially optimized geometry for each state $\left(\mathrm{S}_{1}^{\min }\right)$. Energies of the electronic ground $\left(S_{0}\right)$ and second $\left(S_{2}\right)$ excited state are also given with respect to the $2 B 7(C)$ ground state energy. Oscillator strengths are given in parenthesis. Calculations were performed at the SCS$A D C(2) /$ aug-cc-pVDZ//ADC(2)/cc-pVDZ level for the $n \pi^{*}$ states and at the SCS-ADC(2)/augcC-pVDZ level for the $\pi$ Ryd state.

\begin{tabular}{lllllllll}
\hline \multicolumn{7}{c}{ Adiabatic Excitation Energies $(\mathrm{eV})$} \\
\hline State & $\pi$ Ryd & $\mathrm{n}_{1} \pi_{1}^{*}$ & $\mathrm{n}_{2} \pi_{2}^{*}$ & $\mathrm{n}_{3} \pi_{3}^{*}$ & $\mathrm{n}_{4} \pi_{4}^{*}$ & $\mathrm{n}_{5} \pi_{5}^{*}$ & $\mathrm{n}_{6} \pi_{6}^{*}$ & $\mathrm{n}_{7} \pi_{7}^{*}$ \\
\hline $\mathrm{S}_{0}$ & 0.10 & 1.84 & 1.92 & 1.82 & 2.44 & 1.72 & 2.04 & 1.41 \\
$\mathrm{~S}_{1}^{\text {min }}$ & 4.68 & 3.82 & 4.11 & 3.86 & 4.51 & 3.79 & 4.20 & 3.51 \\
& $(0.0096)$ & $(0.0007)$ & $(0.0004)$ & $(0.0011)$ & $(0.0006)$ & $(0.0009)$ & $(0.0005)$ & $(0.0015)$ \\
$\mathrm{S}_{2}$ & 5.21 & 5.70 & 5.27 & 5.12 & 5.86 & 5.32 & 5.29 & 4.87 \\
& $(0.0009)$ & $(0.0811)$ & $(0.0901)$ & $(0.0888)$ & $(0.1116)$ & $(0.1017)$ & $(0.0861)$ & $(0.0892)$ \\
\hline
\end{tabular}


a)

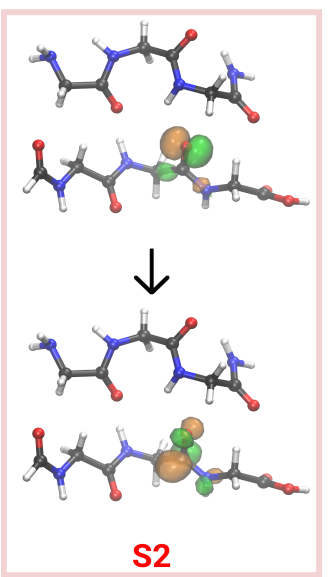

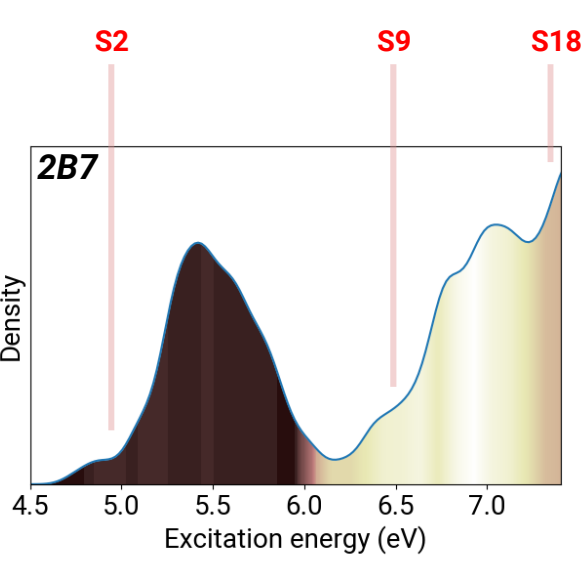
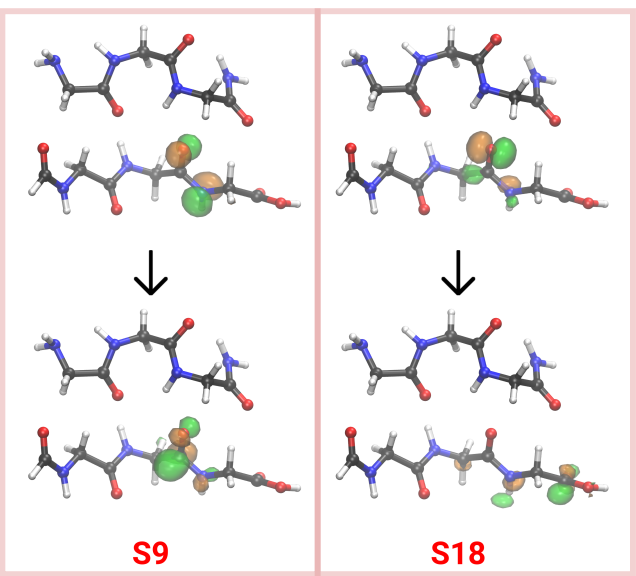

b)
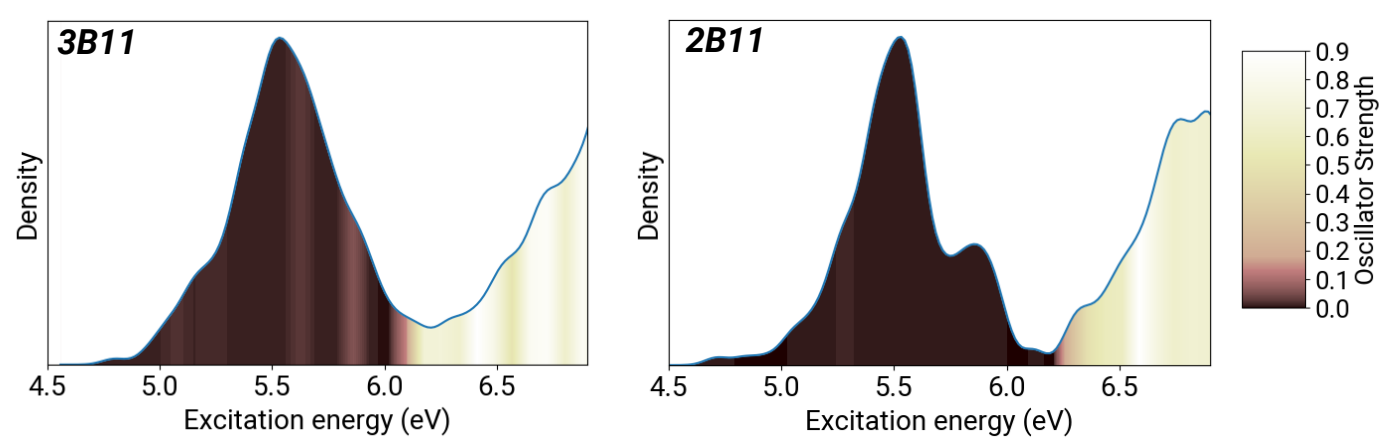

Figure S1: a) Density of excited states (DoES) computed using 30 randomly sampled configurations of 2B7. Dominant natural transition orbital (NTO) pairs of selected electronic transitions are shown for a selected 2B7 geometry. Red lines indicate the approximate energy location of the selected transition b) DoES obtained from 22 configurations of $3 B 11$ and 15 configurations of 2B11. For all DoES the color scale indicates the average oscillator strength. 

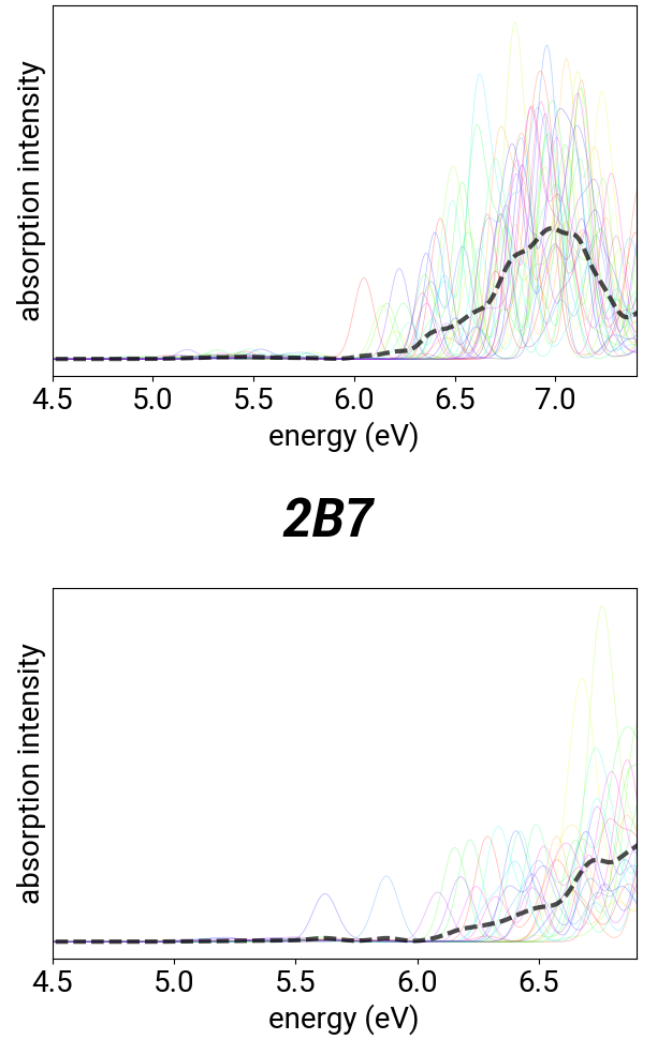

$3 B 11$

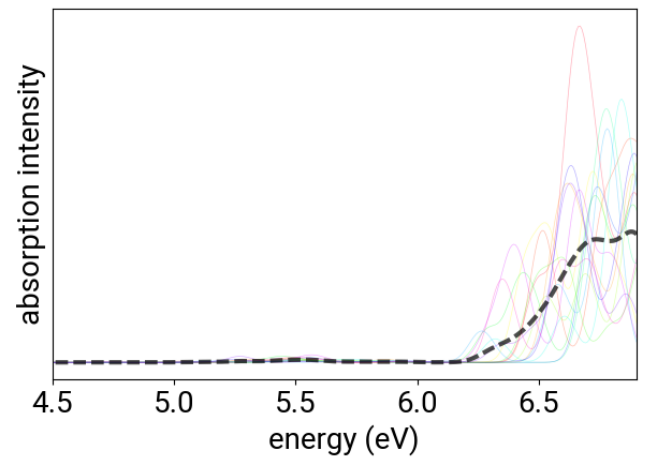

$2 B 11$

Figure S2: UV absorption spectra (thick dashed line) generated averaging spectra from 30 randomly sampled configurations of parallel $\mathbf{2 B} 7,22$ configuration of $\mathbf{3 B} 11$ and 15 configuration of 2B11. Each transition (thin lines) has been broadened by a Gaussian function with $\sigma=$ $0.05 \mathrm{eV}$. Excitation energies have been computed at the ADC(2)/cc-pVDZ level. 


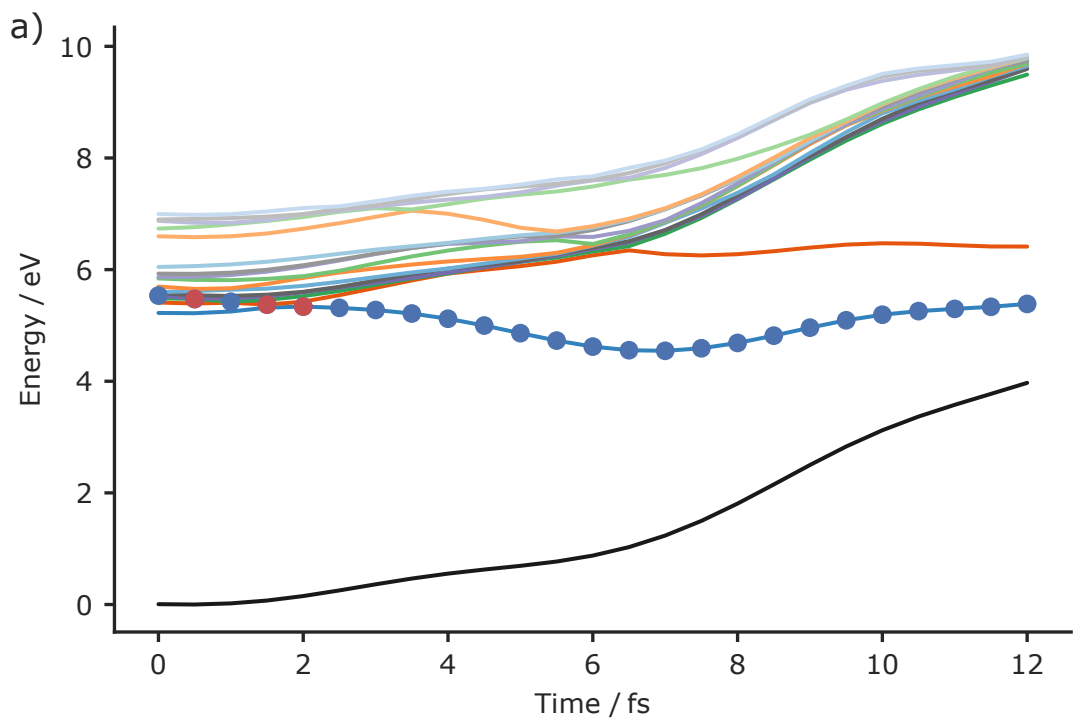

b)
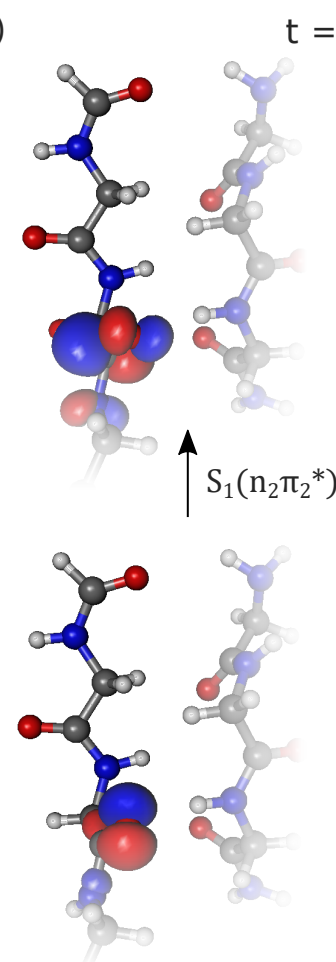

$\mathrm{t}=0 \mathrm{fs}$
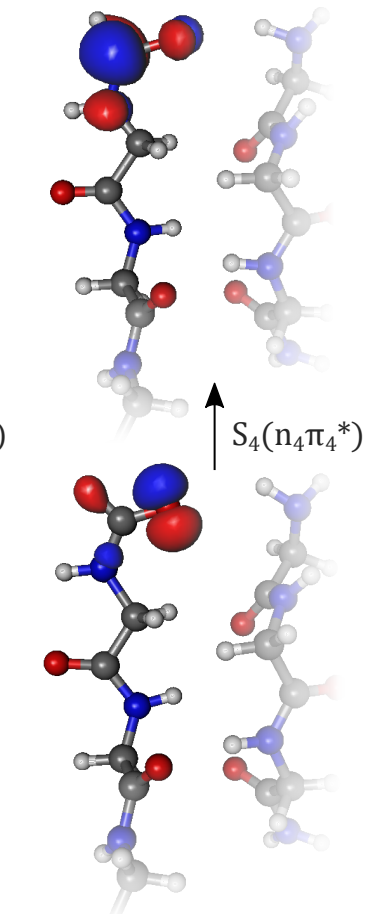

c)

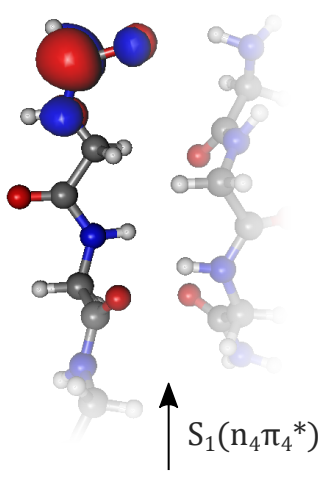

$\mathrm{t}=12 \mathrm{fs}$

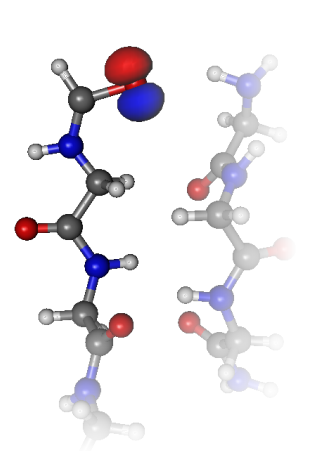

Figure S3: Time dependence of the potential energy of the ground and lowest 20 excited electronic states of 3B11 along a selected ADC(2)/cc-pVDZ nonadiabatic trajectory (b). Fragment of 3B11 with NTO pairs describing the excitation to the lowest excited state $\left(S_{1}\right)$ and the initially excited state $\left(S_{4}\right)$ at the begining of the trajectory (a) and to $S_{1}$ and $S_{2}$ at the end of the trajectory (c). The photoexcited $\mathrm{n} \pi^{*}$ state and the bright $\pi \pi^{*}$ state with excitation localized at the same amide group are quickly stabilized from the higher manifold. 


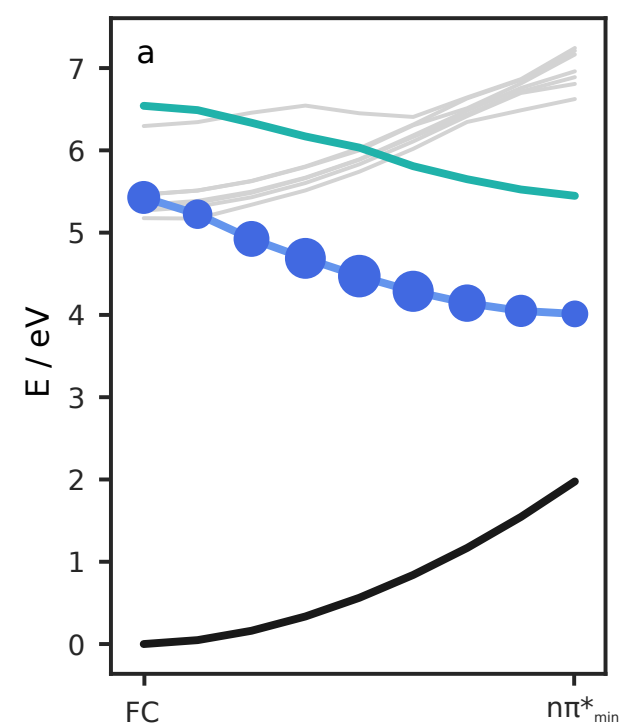

LIIC

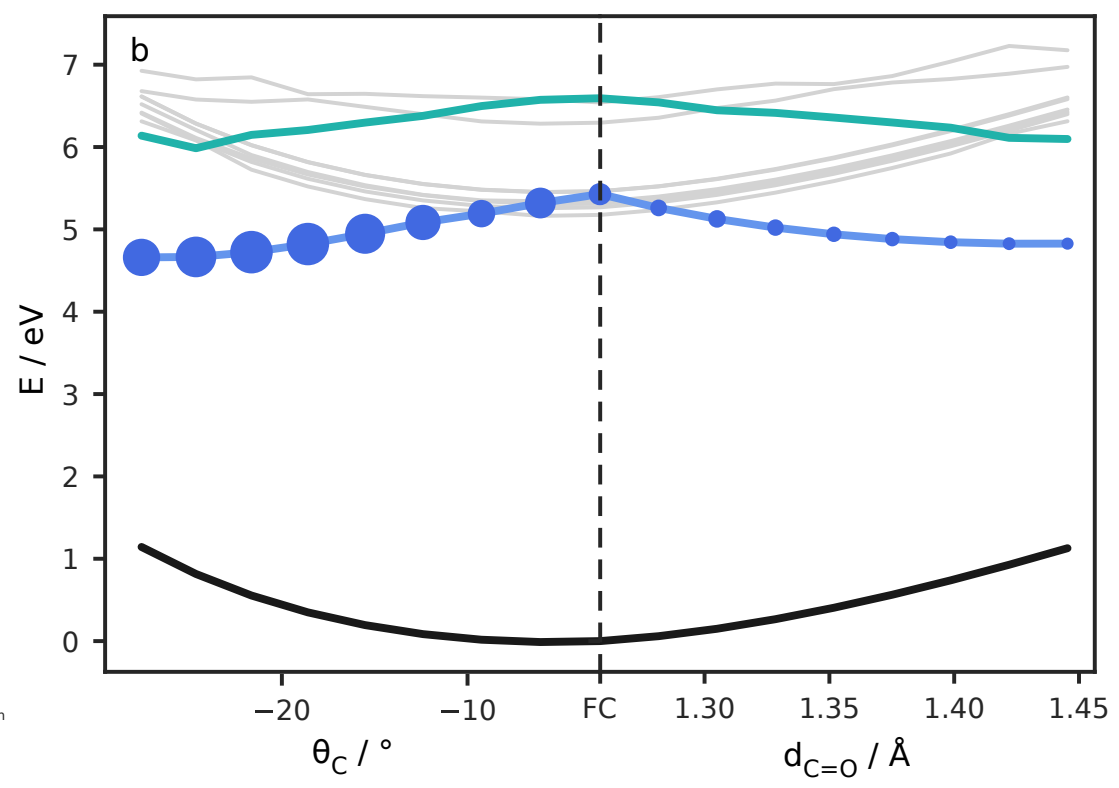

Figure S4: a) Relative ADC(2)/cc-pVDZ energies (in eV) of the ground (black), $\mathrm{n}_{2} \pi_{2}^{*}$ (blue), $\pi_{2} \pi_{2}^{*}$ (green) and 8 other excited states (gray) of 2B7 along the linearly interpolated path (LIP) describing the readjustment from the reference Franck-Condon geometry to the partially optimized $\mathrm{n}_{2} \pi_{2}^{*}$ state minimum. b) The effects of decoupling the deplanarization of the peptide bond $\theta_{C}\left(\mathrm{~N}_{i+1} \mathrm{C}_{i}^{\alpha} \mathrm{O}_{i} \mathrm{C}_{i}\right)$ from the $(\mathrm{C}=\mathrm{O})_{2}$ stretching motion are shown on the left and right panel, respectively. The coordinate dependence of the oscillator strengths of the $n_{2} \pi_{2}^{*}$ state is indicated by blue circles of different areas. 

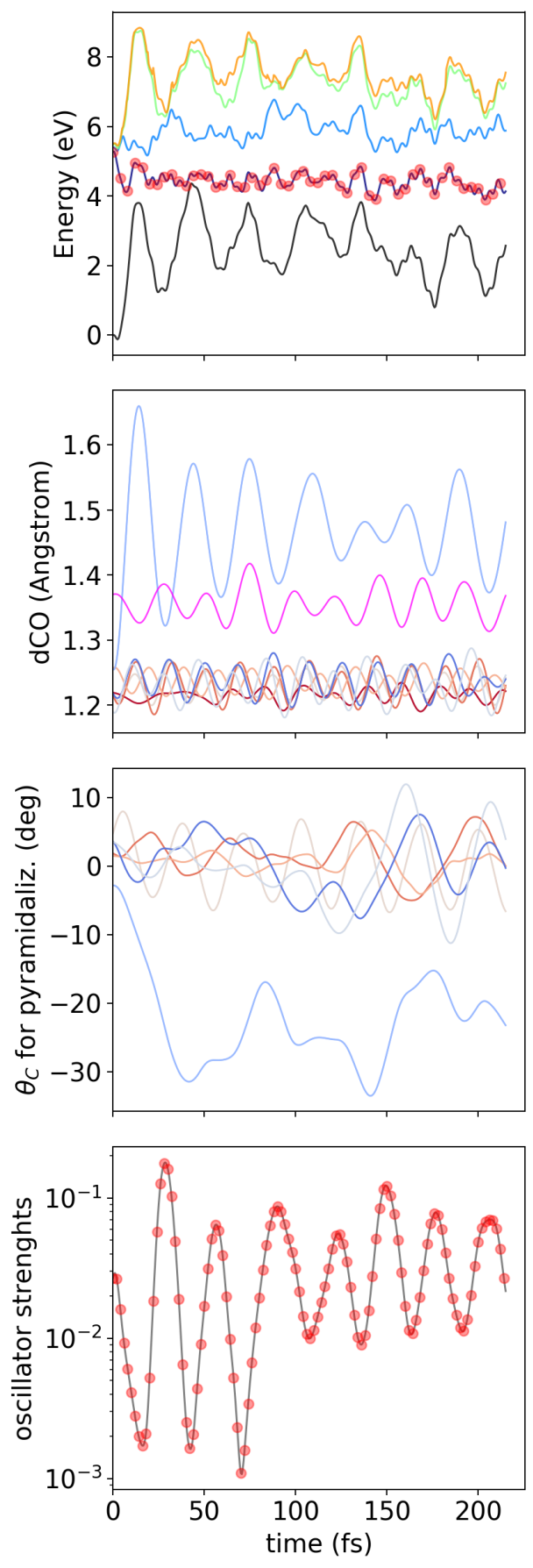

Figure S5: Panels from top to bottom: a) Time evolution of the potential energies of the electronic ground state $\left(\mathrm{S}_{0}\right.$, black curve $)$ and the first four excited electronic states $\left(\mathrm{S}_{1}-\mathrm{S}_{4}\right.$, coloured) for a selected nonadiabatic trajectory of $\mathbf{2 B} 7$ not deactivating to the ground state. The red dots indicate the populated state at a given time. b)Time evolution of the $\mathrm{C}=\mathrm{O}$ bond distances in the system. The electronic excitation is localized on the $\mathrm{C}=\mathrm{O}$ group showing large oscillations. The dependence of the potential energy of the ground state on the elongation of the $C=0$ bond length is visible. c) Time variation of the pyramidalization angle of the carbonyl carbon atoms, $\theta(C)$. d) Time variation of the oscillator strength of the currently populated electronic state. Calculations have been performed at the ADC(2)/cc-pVDZ level. For details, consult the Computational Methods section. 

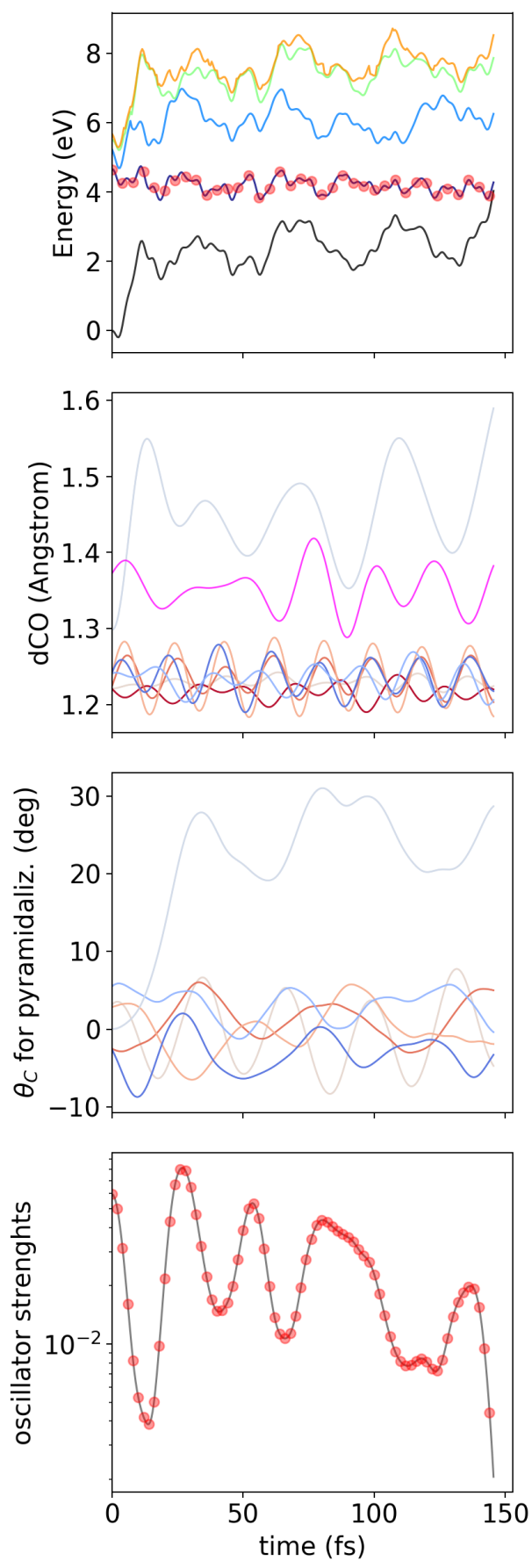

Figure S6: Panels from top to bottom: a) Time evolution of the potential energies of the electronic ground state $\left(\mathrm{S}_{0}\right.$, black curve) and the first four excited electronic states $\left(\mathrm{S}_{1}-\mathrm{S}_{4}\right.$, coloured) for a selected nonadiabatic trajectory of $\mathbf{2 B} 7$ deactivating to the ground state. The red dots indicate the populated state at a given time. b)Time evolution of the $\mathrm{C}=\mathrm{O}$ bond distances in the system. The electronic excitation is localized on the $\mathrm{C}=\mathrm{O}$ group showing large oscillations. The dependence of the potential energy of the ground state on the elongation of the $\mathrm{C}=0$ bond length is visible. c) Time variation of the pyramidalization angle of the carbonyl carbon atoms, $\theta(C)$. d) Time variation of the oscillator strength of the currently populated electronic state. Calculations have been performed at the ADC(2)/cc-pVDZ level. For details, consult the Computational Methods section. 


\section{S2.2 Structural fluctuations and the role of termini}

Static calculations and nonadibatic dynamics simulations revealed that deplanarization $\left(\left|\theta_{C}\right|\right)$ and extension of $\mathrm{CO}$ bonds $\left(d_{C O}\right)$ affect the excitation energies and oscillator strengths (see Figures 3 and 4). Here we look for the frequency of such deformation in our previous 2Y3J crystal ab initio Molecular Dynamics (AIMD). ${ }^{2}$ We are also concerned with the role of the $\mathrm{H}$-bonded termini. Specifically we are interested in whether the excitation energies of $\mathrm{n} \pi^{*}$ states localized on the termini are significantly redshifted with respect to those localized on inner groups. These two issues are connected as deplanarization and CO bond extension are facilitated by $\mathrm{H}$-bond interactions.

2Y3J has 6 residues, and correspondingly 6 carbonyl units (and $7 \mathrm{CO}$ bonds) that were numbered from the $\mathrm{N}$ - to the C-terminus as $j=0 \ldots 4$, while $\mathrm{CO}$ on the $\mathrm{C}$-terminus are marked $j=5$ (dominantly $\mathrm{C}=\mathrm{O}$ ) or $j=56$ (mostly single-bonded $\mathrm{C}-\mathrm{O}$ ). The analysis of the long $2 \mathrm{Y} 3 \mathrm{~J}$ crystal AIMD with eight 2 Y3 $\mathrm{J}$ chains per unit cell and $\approx 45 \mathrm{~K}$ frames shows that structures with $\left|\theta_{C}\right| \gtrsim 10$ and $d_{C O} \gtrsim 1.28$ are found. This is evident from the distributions shown in Figures S7, where a non-negligible number of frames (see color scale) corresponds to geometries with deplanarized amide groups and/or extended $\mathrm{CO}$ bonds.

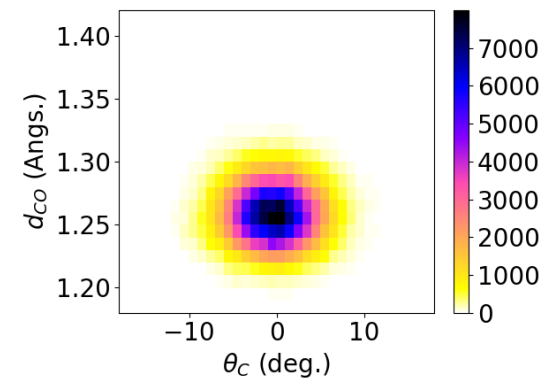

$\mathrm{j}=3$

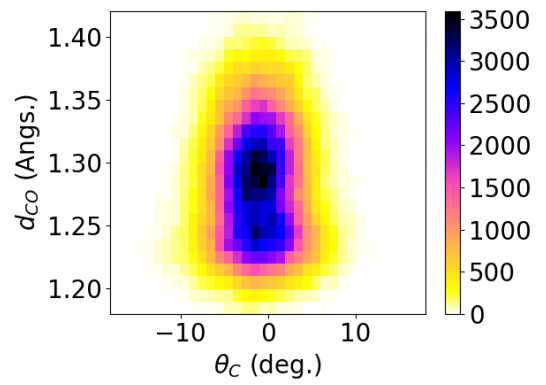

$j=5$

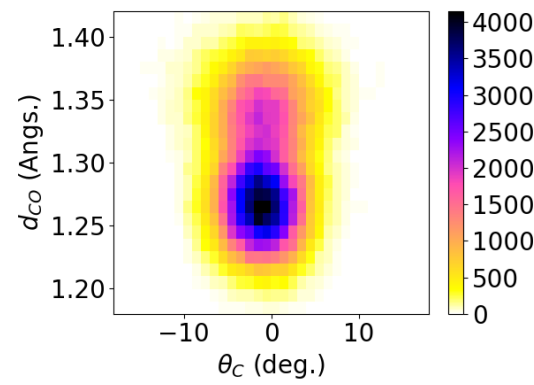

$j=56$

Figure S7: Heatmaps (2D histogram) distributions of $\theta_{C}$ (deg.) vs. $d_{C O}$ bond length (Angstrom), in the 2Y3J AIMD for a central residue $(j=3)$ and a two terminal COs $(j=5$ and $j=56)$. $\theta_{C}$ (deg.) is defined as a dihedral angle $\left(\mathrm{N}, \mathrm{C}_{\alpha_{j}}, \mathrm{O}, \mathrm{C}_{j}\right)$ for $j \leq 4$ and $\left(\mathrm{N}, \mathrm{O}, \mathrm{O}, \mathrm{C}_{j}\right)$ for $j \geq 5$ 
From the 2Y3J chains we then constructed a model system, denoted B1B2, which contains the hydrogen-bonded fragment of two $\beta$-strands. Part of the residues was removed and the model was capped with $\mathrm{NH}_{2}$ groups. The model is shown in Figure S8.

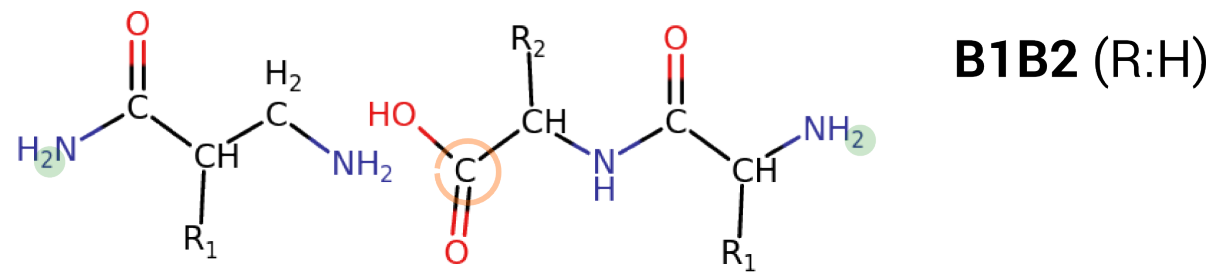

Figure S8: Model system B1B2 obtained from two nearby chains of 2Y3J hexapeptide in the crystal structure. Nearby chain are connected with the salt bridge and part of the residues were dropped. The carbon atom of the $\mathrm{COOH}$ group is marked with an an orange circle, while green halos mark the capping $\mathrm{NH}_{2}$ groups.

Vertical excitation energies and oscillator strengths were computed for a set of B2B3 geometries. Figure S9 shows the densities of excited states (DoES) computed using the ADC(2)/cc-pVDZ (top), SCS-ADC(2)/aug-cc-pVDZ (middle) and SOC-ADC(2)/aug-cc-pVDZ (bottom) methods. It can be seen that there is no significant gap in the DoES between the $\mathrm{n} \pi^{*}$ states localized on the hydrogen bonded termini and those localized on the inner carbonyl groups. States localized on the termini are slightly redshifted but the effect is relatively small. This can be inferred also by comparing the excitation energies of B1B2 (Figure S9 top panel) and those of 2B7, 2B11 and 3B11 (Figure S1) computed using the same electronic structure method, that is $A D C(2) / c c-p V D Z$. Note that in case of B1B2 we have sampled in a target way deplanarized structures whose excitation energies are expected to be redshifted, while $\mathbf{2 B 7}$, 2B11 and 3B11 were randomly sampled. The choice of the electronic structure method and basis set has a quite large effect. The DoES computed with SCS-ADC/aug-cc-pVDZ is redshifted by $\sim 0.4 \mathrm{eV}$ with respect to the ADC(2)/cc-pVDZ DoES, and the one computed with SOC-ADC(2)/aug-cc-pVDZ by around $0.2 \mathrm{eV}$ The addition of diffuse functions noticeably increases the oscillator strengths via Rydberg-valence mixing (see middle panel Fig. S9). While Rydberg-valence interactions may facilitate the excitation in the near UV, the computational demands of the SCS-ADC(2)/aug-cc-pVDZ method are such that it was not possible to perform a complete static and dynamics analysis at that level of theory. 
a)

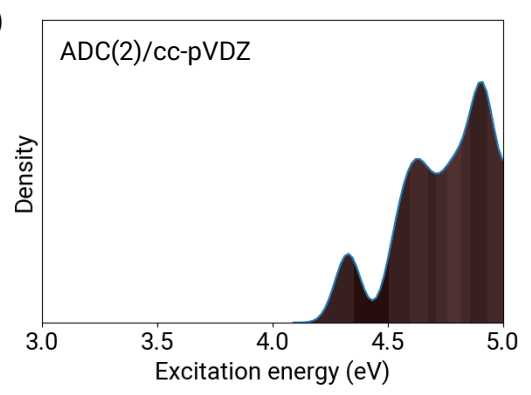

S1 $\quad$ S2 $\quad$ S3
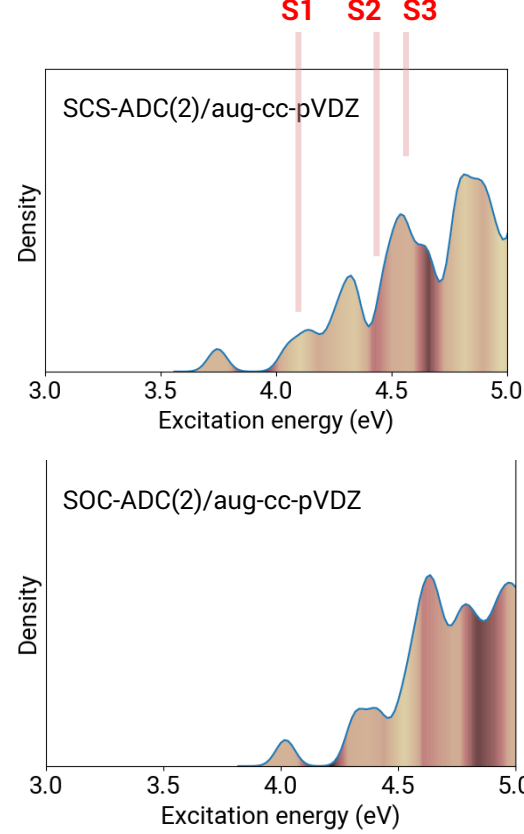

b)
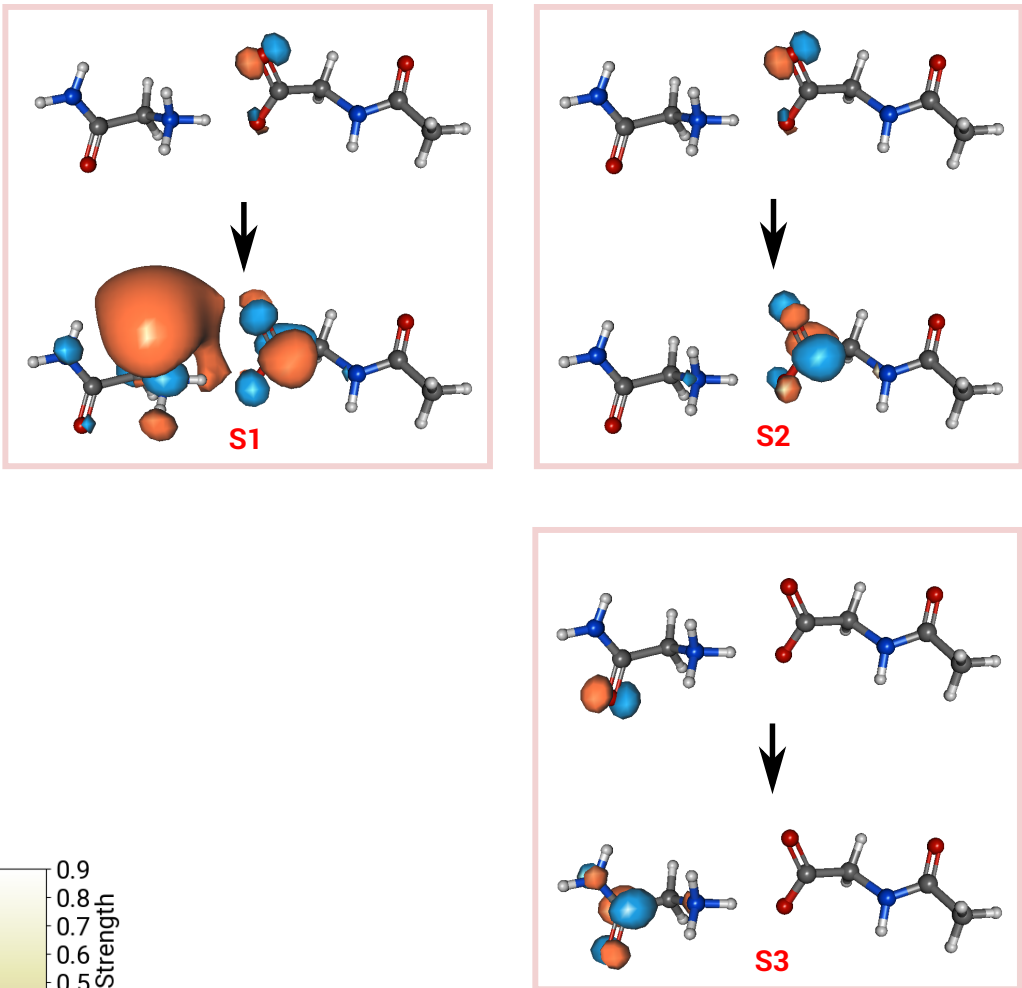

Figure S9: a) Density of excited states computed using 14 selected highly deplanarized configurations of B1B2. Calculations were performed with $A D C$ (2)/cc-pVDZ (top), SCS-ADC(2)/augcc-pVDZ (middle) and SOC-ADC(2)/aug-cc-pVDZ (bottom). b) Dominant natural transition orbital (NTO) pairs of electronic transitions are shown for a selected geometry at the SCS$A D C(2) / a u g-c c-p V D Z$ level of theory. Red lines on the corresponding panel in (a) indicate the approximate energy location of the selected transition. 


\section{S3 Cartesian coordinates of the reference $2 \mathrm{B7}(\mathrm{C})$ structure}

51

\# 2BC7(C) from 2Y3J structure

$\mathrm{H} \quad 0.0000000 \quad 0.0000000$

$\begin{array}{lll}\mathrm{N} & 0.0000000 & 0.0000000\end{array}$

0.0000000

$\begin{array}{lll}\text { C } & 1.2773601 & 0.0000000\end{array}$

1.0153460

C $\quad 1.2025642-1.0803159$

1.7736663

2.8827115

$0.2174042-1.1206722$

3.6605502

$\mathrm{H} \quad 1.5510343 \quad 0.9533542$

2.2389295

$2.2819439-1.9130790$

2.9853964

$2.5527241-2.7117443$

4.1981942

$3.8505248-2.1878740$

4.8692728

$4.8487506-1.9434037$

4.1410359

3.8549146

$-2.0297871$

6.2222362

5.0559800

$-1.4509892$

6.8881543

5.2179200

$-2.0915376$

8.3009464

4.2949240

$-2.0837731$

9.1513927

5.0874371

$-0.3508535$

6.8495547

6.4347018

$-2.6844644$

8.5069308

6.6494282

$-3.3332756$

9.2744926

4.9525659

$-0.5253686$

$-0.9845894$

4.9256175

$-1.2143869$

$-0.1096474$

3.9266287

$-1.2479954$

0.6482254

6.0590835

$-1.9509624$

0.0999870

6.2919689

$-2.7150761$

1. 3391207

7.6433932

$-2.3165235$

1.9862440

8.6614976

$-2.1998167$

1. 2540089

7.6640198

$-2.1538414$

3.3392114

8.9253593

$-1.8103360$

4.0310941

9.0315228

$-2.5605151$

5.3812477

8.0108046

$-2.9263712$

6.0194565

9. 1264944

$-0.7274925$

4.1194675

10.3162314

$-2.8006441$

5.8014575

10.6339476

$-3.7870357$

6.8616327

11. 2097607

$-3.1741987$

8.1683830

12. 3051961

$-2.5970260$

8.2127626

$-4.5028005$

6.4846698

11.3766978

$-3.4531787$

9. 2345369

$-0.8799378$

$-0.1687248$

1.4965178

2.0618704

$-0.2519805$

1. 0442845

3. 0562776

$-1.7809528$

2. 3070137

1.6827465

$-2.6557452$

4.8733535

2.7255326

$-3.7747180$

3.9219594

2.9627443

$-2.1196018$

6.7429229

5.9109221

$-1.7886832$

6.2767265

7.0758518

$-2.7133131$

7.6979192

5.4458708

$-2.5526737$

2.0280533

6.7732631

$-2.1791025$

3.8686397

9.7352085

$-2.1936400$

3.3879564

11.0796122

$-2.5424444$

5.1505140

9.6946800

$-4.3049793$

7.1074725

6.8540612

$-1.8218538$

$-0.5527054$

6.3600408

$-3.8014957$

1.1082858

$\mathrm{H} \quad 10.9398738$

$-3.1325161$

10.1154717 


\section{References}

[1] Colletier, J.-P.; Laganowsky, A.; Landau, M.; Zhao, M.; Soriaga, A. B.; Goldschmidt, L.; Flot, D.; Cascio, D.; Sawaya, M. R.; Eisenberg, D. Molecular basis for amyloid- $\beta$ polymorphism. Proc. Nat. Acad. Sci. U.S.A. 2011, 108, 16938-16943.

[2] Pinotsi, D.; Grisanti, L.; Mahou, P.; Gebauer, R.; Kaminski, C. F.; Hassanali, A.; Kaminski Schierle, G. S. Proton Transfer and Structure-Specific Fluorescence in Hydrogen Bond-Rich Protein Structures. J. Am. Chem. Soc. 2016, 138, 3046-3057.

[3] Hutter, J.; Iannuzzi, M.; Schiffmann, F.; VandeVondele, J. cp2k: atomistic simulations of condensed matter systems. WIREs Comput. Mol. Sci. 2014, 4, 15-25.

[4] Goedecker, S.; Teter, M.; Hutter, J. Separable dual-space Gaussian pseudopotentials. Phys. Rev. B 1996, 54, 1703-1710.

[5] Becke, A. D. Density-functional exchange-energy approximation with correct asymptotic behavior. Phys. Rev. A 1988, 38, 3098-3100.

[6] Lee, C.; Yang, W.; Parr, R. G. Development of the Colle-Salvetti correlation-energy formula into a functional of the electron density. Phys. Rev. B 1988, 37, 785-789.

[7] Grimme, S.; Antony, J.; Ehrlich, S.; Krieg, H. A consistent and accurate ab initio parametrization of density functional dispersion correction (DFT-D) for the 94 elements H-Pu. J. Chem. Phys. 2010, 132, 154104.

[8] Byrd, R.; Lu, P.; Nocedal, J.; Zhu, C. A limited memory algorithm for bound constrained optimization. SIAM J. Sci. Comput. 1995, 16, 1190-1208.

[9] VandeVondele, J.; Krack, M.; Mohamed, F.; Parrinello, M.; Chassaing, T.; Hutter, J. Quickstep: Fast and accurate density functional calculations using a mixed Gaussian and plane waves approach. Comput. Phys. Commun. 2005, 167, $103-128$.

[10] Bussi, G.; Donadio, D.; Parrinello, M. Canonical sampling through velocity rescaling. J. Chem. Phys. 2007, 126, 014101.

[11] Schirmer, J. Beyond the random-phase approximation: A new approximation scheme for the polarization propagator. Phys. Rev. A 1982, 26, 2395-2416. 
[12] Dreuw, A.; Wormit, M. The algebraic diagrammatic construction scheme for the polarization propagator for the calculation of excited states. WIREs Comput. Mol. Sci. 2015, 5, 82-95.

[13] Dunning, T. H. Gaussian basis sets for use in correlated molecular calculations. I. The atoms boron through neon and hydrogen. J. Chem. Phys. 1989, 90, 1007-1023.

[14] Grimme, S. Improved second-order Møller-Plesset perturbation theory by separate scaling of parallel- and antiparallel-spin pair correlation energies. J. Chem. Phys. 2003, 118, 9095-9102.

[15] Hellweg, A.; Grün, S. A.; Hättig, C. Benchmarking the performance of spin-component scaled CC2 in ground and electronically excited states. Phys. Chem. Chem. Phys. 2008, 10, 4119-4127.

[16] Furche, F.; Ahlrichs, R.; Hättig, C.; Klopper, W.; Sierka, M.; Weigend, F. Turbomole. WIREs Comput. Mol. Sci. 2014, 4, 91-100.

[17] TURBOMOLE V7.0 2015, a development of University of Karlsruhe and Forschungszentrum Karlsruhe $\mathrm{GmbH}$, 1989-2007, TURBOMOLE $\mathrm{GmbH}$, since 2007; available from http://www. turbomole. com.

[18] Tully, J. C. Molecular dynamics with electronic transitions. J. Chem. Phys. 1990, 93, 1061-1071.

[19] Hammes-Schiffer, S.; Tully, J. C. Proton transfer in solution: Molecular dynamics with quantum transitions. J. Chem. Phys. 1994, 101, 4657-4667.

[20] Sapunar, M.; Piteša, T.; Davidović, D.; Došlić, N. Highly Efficient Algorithms for CIS Type Excited State Wave Function Overlaps. J. Chem. Theory Comput. 2019, 15, 3461-3469.

[21] Plasser, F.; Crespo-Otero, R.; Pederzoli, M.; Pittner, J.; Lischka, H.; Barbatti, M. Surface Hopping Dynamics with Correlated Single-Reference Methods: 9H-Adenine as a Case Study. J. Chem. Theory Comput. 2014, 10, 1395-1405.

[22] Chaiwongwattana, S.; Sapunar, M.; Ponzi, A.; Decleva, P.; Došlić, N. Exploration of excited state deactivation pathways of adenine monohydrates. J. Phys. Chem. A 2015, 119, 10637-10644.

[23] Mališ, M.; Došlić, N. Nonradiative relaxation mechanisms of UV excited phenylalanine residues: A comparative computational study. Molecules 2017, 22, 493.

[24] Granucci, G.; Persico, M.; Toniolo, A. Direct semiclassical simulation of photochemical processes with semiempirical wave functions. J. Chem. Phys. 2001, 114, 10608-10615. 
[25] Granucci, G.; Persico, M. Critical appraisal of the fewest switches algorithm for surface hopping. J. Chem. Phys. 2007, 126, 134114. 